Revista del Centro de Investigación de la Universidad La Salle

Vol. 13, No. 51, enero-junio, 2019: 67-80

DOI: http://dx.doi.org/10.26457/recein.v13i51.2200

\title{
Primer abordaje para la propagación de Anaplasma marginale (MEX-31-096) en células de garrapata $R \mathbf{m}$-sus
}

\section{First approach for the propagation of Anaplasma marginale (MEX-31-096) in Rm-sus tick cells}

Mayra Elizeth Cobaxin-Cardenas ${ }^{1}$

CENID Salud Animal e Inocuidad, INIFAP (México)

Hugo Aguilar Díaz

CENID Salud Animal e Inocuidad, INIFAP (México)

Perla Olivares Avelino

CENID Salud Animal e Inocuidad, INIFAP (México)

Elizabeth Salinas-Estrella

CENID Salud Animal e Inocuidad, INIFAP (México)

Jesús Francisco Preciado-de la Torre

CENID Salud Animal e Inocuidad, INIFAP (México)

Rosa Estela Quiroz-Castañeda

CENID Salud Animal e Inocuidad, INIFAP (México)

Itzel Amaro-Estrada

CENID Salud Animal e Inocuidad, INIFAP (México)

${ }^{1}$ Email: mayracobaxin@gmail.com

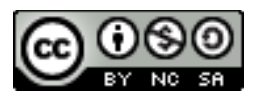

Revista del Centro de Investigación. Universidad La Salle por Dirección de Posgrado e Investigación. Universidad La Salle Ciudad de México se distribuye bajo una Licencia Creative Commons Atribución- 
Cobaxin-Cardenas, M; Aguilar Díaz, H.; Olivares Avelino, P.; Salinas-Estrella, E.; Preciado-de la Torre, J.; Quiroz-Castañeda, R.; Amaro-Estrada, I.; Cossío-Bayúgar, R.; Rodríguez-Camarillo, S.

\section{Raquel Cossío-Bayúgar \\ CENID Salud Animal e Inocuidad, INIFAP (México) \\ Sergio Rodríguez-Camarillo \\ CENID Salud Animal e Inocuidad, INIFAP (México)}

Recibido: 06 de julio de 2019

Aceptado: 22 de noviembre de 2019

Publicado: 13 de diciembre de 2019

\section{Resumen}

Anaplasma marginale es una bacteria intraeritrocítica transmitida por garrapatas que causa la anaplasmosis bovina. Una limitante para el estudio de este microorganismo en México ha sido la dificultad para cultivarla in vitro. A la fecha, no se ha reportado el cultivo de aislados o cepas mexicanas de A. marginale, por lo que este es el primer abordaje para establecer la infección y propagación de la cepa MEX-31-096 en células de garrapata Rm-sus, utilizando eritrocitos infectados recién obtenidos como inóculo. La infección se confirmó por microscopia de luz observando el desarrollo de $A$. marginale en las células embrionarias de garrapata Rm-sus a partir del $2^{\text {do }}$ día post inoculación (dpi) y alcanzando el $80 \%$ de células infectadas a los 8 dpi. Asimismo, la amplificación de la región variable del gen mspla por PCR punto final fue positiva. Estos resultados demuestran que A. marginale (MEX-31-096) tiene la capacidad para infectar y propagarse en células Rm-sus, lo que permitirá obtener estos microorganismos bajo condiciones controladas y en menor tiempo facilitando el estudio sobre su fisiología y la interacción patógeno-vector.

Palabras clave: Células de garrapata, Anaplasma marginale, cultivo in vitro 


\section{Abstract}

Anaplasma marginale is an intraerythrocytic bacterium transmitted by ticks that causes bovine anaplasmosis. One of the limitations in the study of this microorganism has been the difficulty to cultivate it in vitro. To date, the cultivation of Mexican strains of $A$. marginale has not been reported. In this work we show the first approach to establish the infection and the propagation of the strain MEX-31-096 in cells of tick's Rm-sus, using as inoculum freshly erythrocytes. The infection was confirmed by light microscopy, observing the development of $A$. marginale in the $\mathrm{Rm}$-sus cells from the $2^{\text {nd }}$ day after inoculation (dpi) and obtaining $80 \%$ of infected cells at day 8 dpi. Likewise, the amplification of the variable region of the mspla gene by PCR endpoint was positive. These results demonstrate that A. marginale (MEX-31-096) has the capacity to infect and propagate in Rm-sus tick cells, which will allow obtaining these microorganisms under controlled conditions and in a shorter time facilitating the study of their physiology and pathogen-vector interaction.

Keywords: Rm-sus cells, Anaplasma marginale, in vitro culture. 
Cobaxin-Cardenas, M; Aguilar Díaz, H.; Olivares Avelino, P.; Salinas-Estrella, E.; Preciado-de la Torre, J.; Quiroz-Castañeda, R.; Amaro-Estrada, I.; Cossío-Bayúgar, R.; Rodríguez-Camarillo, $\mathrm{S}$.

\section{Introducción}

Anaplasma marginale (orden Rickettsiales, familia Anaplasmataceae), es una bacteria intraeritrocítica transmitida por garrapatas que causa la anaplasmosis bovina (Kocan, et. al., 2003), cuyos signos clínicos incluyen, anemia, ictericia, ataxia, pérdida de peso y de producción de leche, aborto y muerte cuando el tratamiento específico no se aplica a tiempo (Quiroz-Castañeda, Amaro-Estrada \& Rodríguez-Camarillo, 2016; Rodríguez, et. al., 2009). Esta enfermedad es enzóotica en México -cuya prevalencia serológica y molecular ha sido superior al 50\% en bovinos de zonas tropicales y subtropicales (Rodríguez, et. al., 2009) - y en la mayoría de los países de América Latina, por la cual se generan grandes pérdidas económicas a la industria ganadera cuando aparecen brotes en el ganado susceptible. De acuerdo con Whitlock (2014) esta enfermedad contribuye a la pérdida de \$300,000 dólares al año para la industria cárnica en Estados Unidos. Las pérdidas son difíciles de estimar en nuestro país, debido a una gran variedad de factores como las razas, la edad, el género, el estado fisiológico, el número de animales afectados y la presencia de otras enfermedades, sin embargo, desde 1999 se hablaba del 26\% de muertes -sólo a causa de la anaplasmosis- de ganado mejorado que era introducido en zonas de elevada prevalencia (Rodríguez, et. al., 2009). En el último informe mensual de la situación zoosanitaria nacional (03/10/2019) se reportan solamente 5 estados de la República Mexicana en situación Libre, 1 en Erradicación y 26 en Control, respecto a la Campaña Oficial de Control de la Garrapata (Servicio Nacional de Sanidad, Inocuidad y Calidad Agroalimentaria, 2019), lo cual sirve de referencia ya que la garrapata es el vector biológico de A. marginale.

Una de las limitaciones en el estudio de A. marginale ha sido la dificultad para cultivarlo in vitro, ya que se necesita de la replicación de este microorganismo y, en ausencia del cultivo se requiere el uso de ganado esplenectomizado, con las respectivas implicaciones en el en el bienestar animal que resultan innecesarias en comparación con la alternativa. Si bien se ha reportado el cultivo de algunos aislados y/o cepas de A. marginale provenientes de los EE. UU y Brasil en células embrionarias de garrapata IDE8 (derivadas de embriones de Ixodes scapularis) (Blouin \& Kocan, 1998; Hammac, et. al., 2013; Munderloh, et. al., 1996; 
Ortiz, et. al., 2000; Silveira, et. al., 2016) a la fecha, no se ha reportado el cultivo in vitro de cepas mexicanas por lo que el objetivo de este trabajo fue determinar si la cepa MEX-31-096 es capaz de infectar y propagarse en una línea celular Rm-sus (derivada de embriones de la garrapata Rhipicephalus microplus) generada en nuestro instituto (Cossío-Bayugar \& MirandaMiranda, 2007).

En este trabajo se reporta por primera vez, la propagación in vitro de A. marginale en células Rm-sus. La bacteria fue capaz de infectar y propagarse en esta línea celular, asimismo, el desarrollo de la bacteria fue similar a lo reportado por otros autores en la línea células IDE8 (Bastos, et. al., 2010; Servicio Nacional de Sanidad, Inocuidad y Calidad Agroalimentaria, 2019; Munderloh, 1996). Este primer abordaje para el cultivo de A. marginale ofrece la oportunidad de estudiar este patógeno en nuestro grupo de investigación ya que la propagación de cepas o aislados mexicanos de A. marginale en células Rm-sus permitirá profundizar en la caracterización de su virulencia, así mismo permitirá estudiar la susceptibilidad a sustancias garrapaticidas o antibióticos cuyo efecto permita dilucidar estrategias de control para la enfermedad ocasionada por este patógeno.

\section{Materiales y Métodos}

\subsection{Preparación del inóculo de A. marginale}

La cepa MEX-31-096 de A. marginale utilizada en este trabajo se aisló de un caso clínico en la localidad de Tizimín, Yucatán, México (Ortiz, et. al., 2000). Para la replicación de la cepa se utilizó un bovino macho (Bos taurus) de 22 meses de edad libre de garrapatas y libre de anticuerpos contra tuberculosis y brucelosis según las normas de las campañas oficiales. El animal fue esplenectomizado e inoculado con la cepa MEX-31-096. Cuando el bovino alcanzó un $17 \%$ de eritrocitos infectados, la sangre fue colectada por punción en la vena yugular y fue procesada como se describió anteriormente (Blouin, et. al., 2000). El número total de eritrocitos se determinó en la cámara de neubauer. 
Cobaxin-Cardenas, M; Aguilar Díaz, H.; Olivares Avelino, P.; Salinas-Estrella, E.; Preciado-de la Torre, J.; Quiroz-Castañeda, R.; Amaro-Estrada, I.; Cossío-Bayúgar, R.; Rodríguez-Camarillo, S.

\subsection{Cultivo de células Rm-sus}

El método de la obtención de los cultivos embrionarios a partir de huevos de garrapata (Rhipicephalus microplus) se realizó de acuerdo a lo descrito (Cossio-Bayugar, R., \& MirandaMiranda, 2007). Las células embrionarias Rm-sus fueron descongeladas del nitrógeno líquido y cultivadas en frascos de $25 \mathrm{~cm}^{2}$, (Corning) en un volumen final de $5 \mathrm{ml}$ de un medio compuesto por una mezcla de Leibovitz's L-15 - MEM (1:1); suplementado con 10\% caldo triptosa fosfato (Sigma), 20\% Suero fetal bovino (Gibco) y antibióticos (penicilina $100 \mathrm{U} / \mathrm{mL}$ y estreptomicina $100 \mu \mathrm{g} / \mathrm{mL}$, (Gibco). Los cultivos se mantuvieron a $32{ }^{\circ} \mathrm{C}$, con una atmosfera de $3 \% \mathrm{CO}_{2}$ y con cambio de medio dos veces por semana. Las células se subcultivaron en un radio 1:2 cuando la monocapa alcanzó una concentración de 4x10 cels/ml. Para los experimentos de infección se utilizaron células en pases entre 92 y 99.

\subsection{Inoculación de las células Rm-sus}

Monocapas confluentes de células Rm-sus se inocularon con $1.2 \times 10^{7}$ eritrocitos infectados con la cepa MEX-31-096. Brevemente, los eritrocitos lavados se resuspendieron en $5 \mathrm{ml}$ de medio Leibovitz's L-15 - MEM (1:1); suplementado con 10\% caldo triptosa fosfato, $20 \%$ Suero fetal bovino) y para que el medio fuese más apropiado para el crecimiento de $A$. marginale, fue adicionado con $0.1 \%$ de $\mathrm{NaHCO}_{3}$ y $10 \mathrm{mM}$ HEPES de acuerdo a lo descrito (Lis, at. al., 2015). Las células inoculadas se mantuvieron en incubación durante veinticuatro horas en las condiciones ya descritas. El primer cambio de medio se realizó a las 24 horas para retirar el inóculo y los cambios de medio subsiguientes se realizaron una vez a la semana.

La propagación de A. marginale se realizó cuando las células alcanzaron el nivel máximo de infección (80-90\%). Para este fin, las células infectadas se cosecharon y se inocularon en monocapas de células no infectadas en un radio 1:2.

\subsection{Monitoreo de la infección}

Los cultivos se monitorearon diariamente por la examinación bajo un microscopio invertido con contraste de fases (OLYMPUS CKX41) con el objetivo de 40X. Se tomaron muestras de 
células periódicamente para realizar improntas que fueron teñidas con Giemsa, las cuales se analizaron en un microscopio de luz convencional $\left(V E L A B^{\circledR}\right.$ VE-BC1) bajo el objetivo de inmersión en aceite.

\subsection{Extracción de DNA genómico y amplificación por PCR}

La extracción de DNA genómico (gDNA) se realizó a partir de células infectadas con $A$. marginale (MEX-31-096), como control positivo se utilizó sangre infectada con A. marginale (MEX-31-096) y como control negativo se utilizaron células no infectadas utilizando un kit comercial (DNeasy Blood \& Tissue Handbook, QIAGEN) de acuerdo a las instrucciones del fabricante. La amplificación del fragmento variable del gen mspl $\alpha$ de A. marginale se realizó por PCR utilizando los oligonucleótidos sentido 5'-GTGCTTATGGCAGACATTTCC-3' y antisentido 5'- CTCAACACTCGCAACCTTGG-3' (Palmer, et. al., 2004) a una concentración de $10 \mathrm{mM}$ y como templado una concentración de 300 ng del gDNA correspondiente y con el siguiente protocolo de amplificación: desnaturalización inicial a $94^{\circ}$ C por 5 minutos, seguidos de 35 ciclos: desnaturalización a $94^{\circ} \mathrm{C}$ por 30 segundos, hibridación a $56^{\circ} \mathrm{C}$ por 45 segundos, extensión a $72^{\circ} \mathrm{C}$ por 45 segundos y un ciclo de extensión final a $72^{\circ} \mathrm{C}$ por 10 minutos. El producto de PCR se sometió a una electroforesis en geles de agarosa al $1 \%(\mathrm{p} / \mathrm{v})$ y se tiñó con SYBR Green para la visualización de los fragmentos amplificados.

\section{Resultados y discusión}

Los primeros indicios de la infección fueron visibles a partir del $5^{\text {to }}$ dpi bajo un microscopio invertido de contraste de fases (Figura 1) observándose un incremento en el tamaño de las células, así como la presencia de gránulos. 
Cobaxin-Cardenas, M; Aguilar Díaz, H.; Olivares Avelino, P.; Salinas-Estrella, E.; Preciado-de la Torre, J.; Quiroz-Castañeda, R.; Amaro-Estrada, I.; Cossío-Bayúgar, R.; Rodríguez-Camarillo, S.

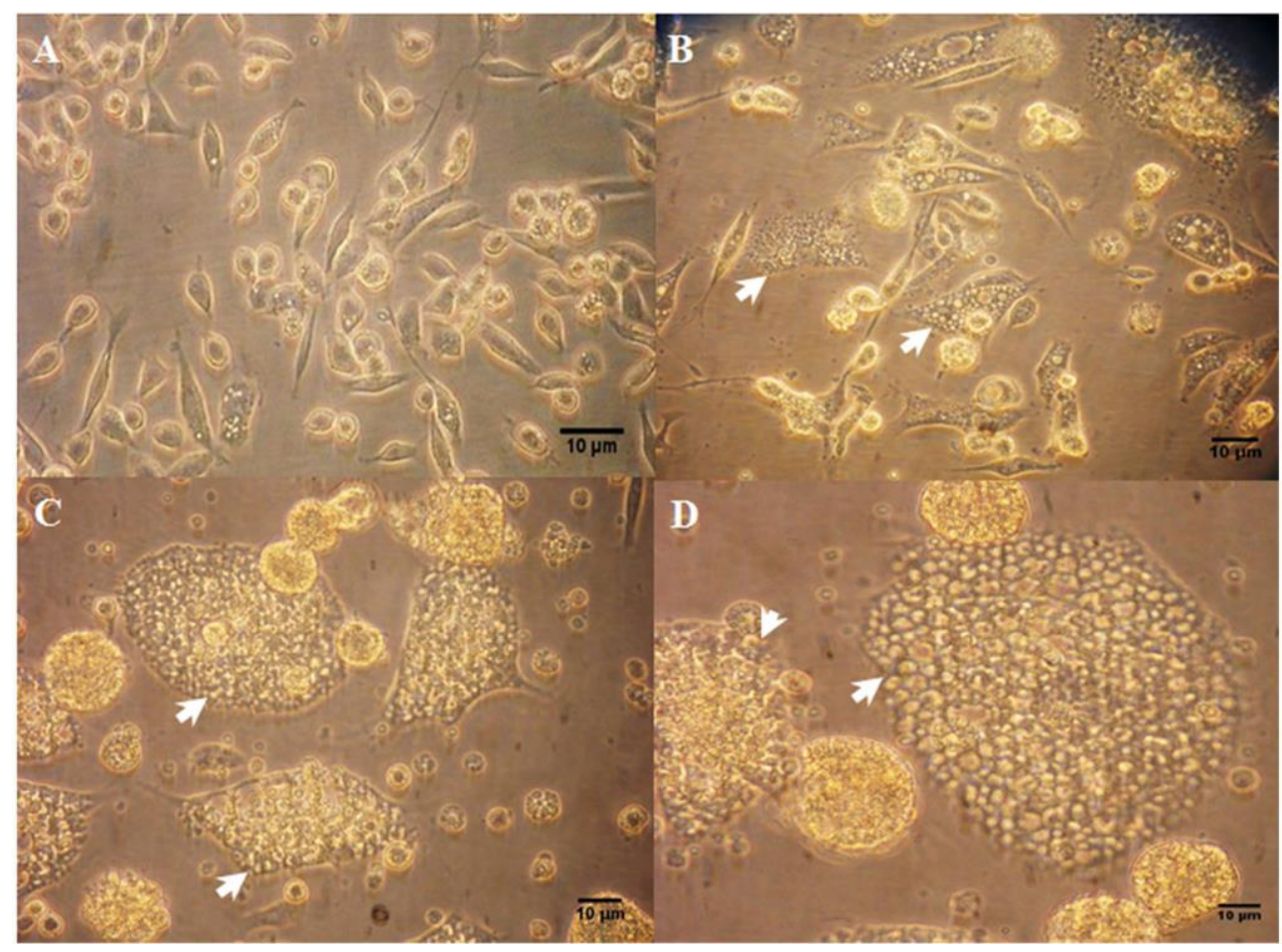

Figura 1. Fotomicrografía de células Rm-sus infectadas con A. marginale (MEX-31-096). A) Células Rm-sus no infectadas, B) Células a los 5 dpi. C y D) Células a los 7 dpi. Se observa un incremento de tamaño en las células (flechas), así como presencia de gránulos sobre la superficie. Las células se visualizaron en un microscopio invertido de contraste de fases con el objetivo 10X (A y B) y 40X (C y D). Barra de calibración $10 \mu \mathrm{m}$.

En contraste a lo observado por microscopía de contraste de fases, en las improntas teñidas con Giemsa las primeras células infectadas se detectaron a partir del $2^{\text {do }}$ dpi, observándose pequeñas colonias intensamente teñidas en el citoplasma (Figura 2, B) y en los días posteriores, estas colonias fueron incrementando en número y tamaño, así como la dislocación del núcleo hacia la membrana en la etapa más avanzada de la infección (Figura 2, C y D). 


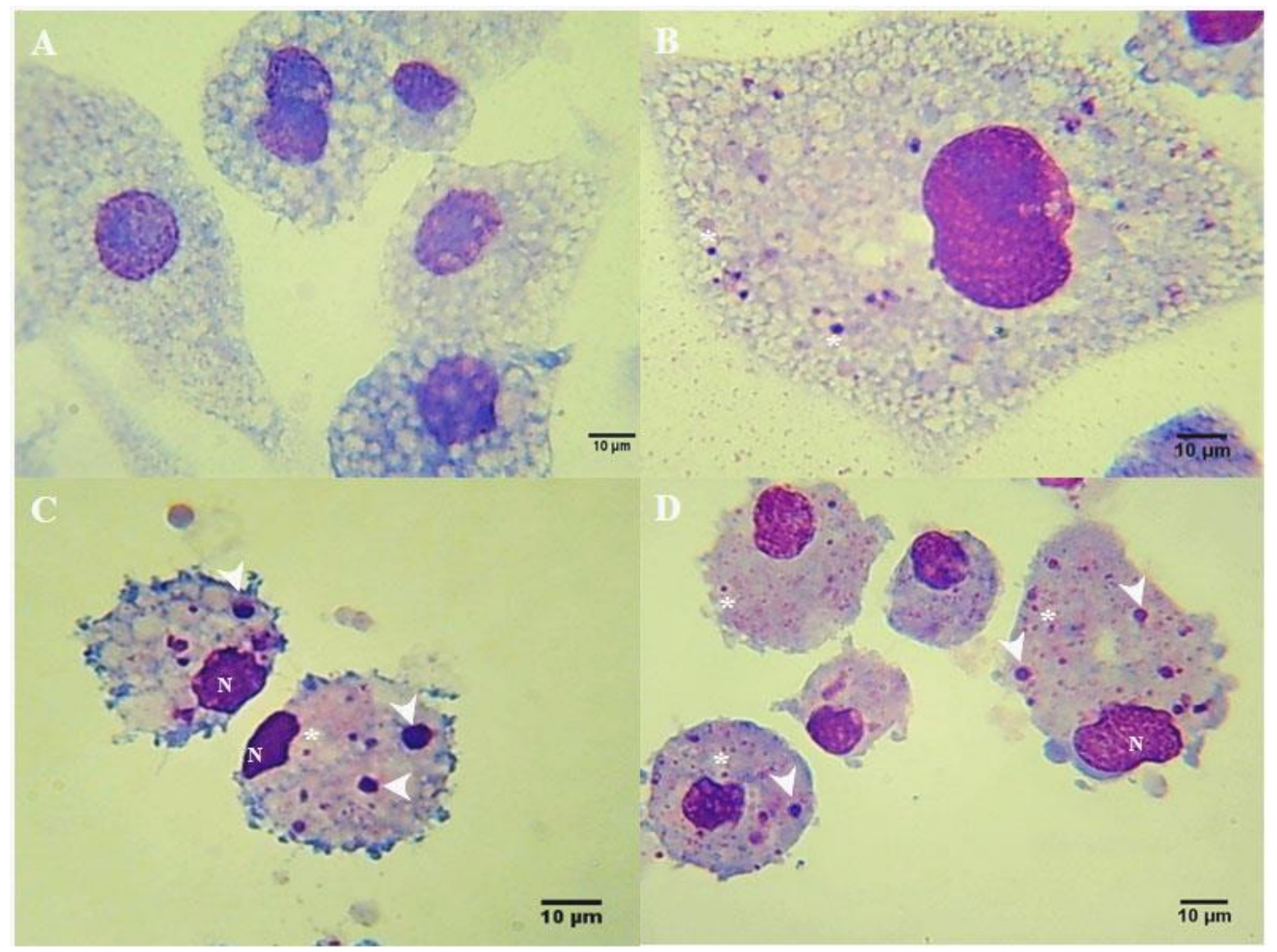

Figura 2. Fotomicrografía de células Rm-sus infectadas con A. marginale (MEX-31-096). A) Células no infectadas. B) Se observan la formación de las primeras colonias de A. marginale en el citoplasma de la célula (asteriscos) ( $\left.2^{\mathrm{do}} \mathrm{dpi}\right)$. C) Las colonias de A. marginale fueron incrementando su tamaño con respecto al tiempo (flechas), además se observa la dislocación del núcleo. D) Células con múltiples colonias de diferentes tamaños se observaron a partir de $4^{\text {to }}$ dpi. Barra de calibración $10 \mu \mathrm{m}$.

En conjunto, las observaciones descritas indican que A. marginale se desarrolla dentro de las células Rm-sus y que estas bacterias son capaces de infectar nuevas células ya que el número de células infectadas se incrementó alcanzando el $80 \%$ a los 8 dpi, a este tiempo las células presentaron daño celular severo (Figura 3B) y en algunos casos se observó lisis celular. Este proceso de desarrollo de A. marginale en las células $\mathrm{Rm}$-sus es similar a lo descrito por otros autores (Blouin, et. al., 1998; Esteves, et. al., 2009). 
Cobaxin-Cardenas, M; Aguilar Díaz, H.; Olivares Avelino, P.; Salinas-Estrella, E.; Preciado-de la Torre, J.; Quiroz-Castañeda, R.; Amaro-Estrada, I.; Cossío-Bayúgar, R.; Rodríguez-Camarillo, S.

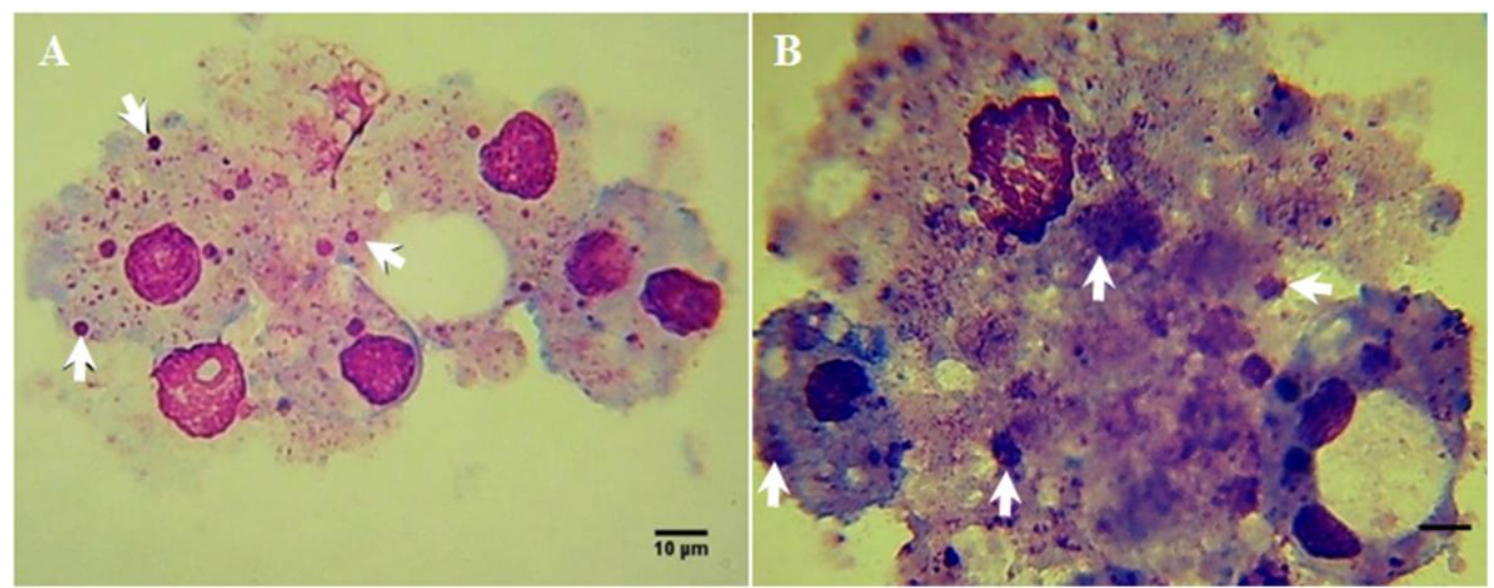

Figura 3. Fotomicrografía de células Rm-sus infectadas con A. marginale (MEX-31-096). A) Célula infectada con A. marginale (flechas) a los 8 dpi. B) Célula infectada a los 14 dpi con colonias grandes de A. marginale (flechas) y daño celular severo. C) Célula infectada a los 21 dpi con en donde se observa lisis celular y las colonias de A. marginale son liberadas al medio extracelular. Barra de calibración $10 \mu \mathrm{m}$.

Adicionalmente al análisis por microscopía, se realizó la amplificación de gen mspla, resultando positiva en los primeros 5 pases (Figura 4 carriles 5 al 9). Sin embargo, en el pase número 6 la amplificación fue negativa para A. marginale (Figura 4 carril 10), y aunque morfológicamente las células presentaron daño celular no fue posible detectar a la bacteria en improntas teñidas con Giemsa (datos no mostrados), lo anterior, podría estar relacionado con la inestabilidad del $\mathrm{pH}$ en el medio de cultivo ya que se ha mostrado que A. marginale es sensible a $\mathrm{pH}$ (10). Por otra parte, la repentina ausencia de la bacteria en los cultivos in vitro ya ha sido reportado por otros autores y una de las posibles causas señaladas está relacionada con el mantenimiento del $\mathrm{pH}$ dentro en la vacuola donde se encuentran las colonias de A. marginale (Silveira, et. al., 2016). 


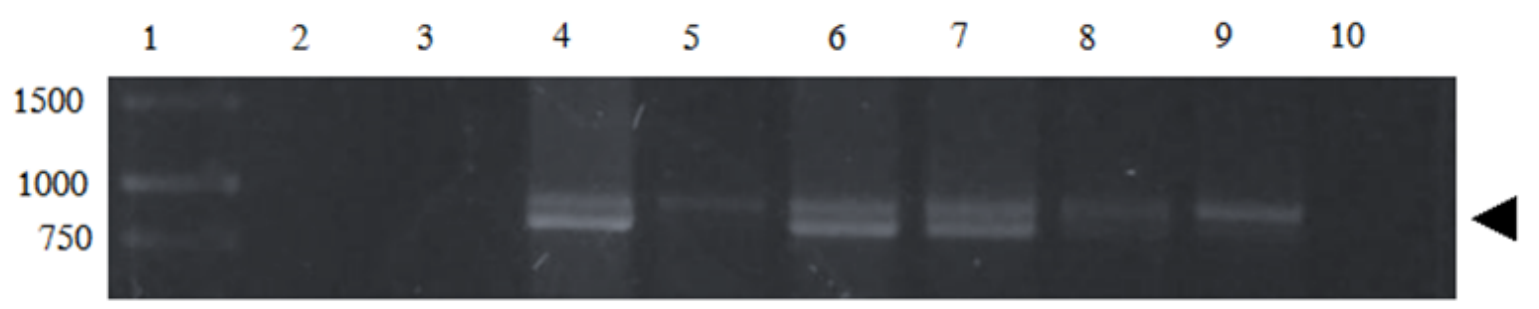

Figura 4. Detección molecular de A. marginale (MEX-31-096) en células Rm-sus infectadas. El gel de agarosa al $1 \%$ teñido con SYBR green muestra un fragmento de $790 \mathrm{pb}$ de la región variable de $m s p 1 \alpha$ amplificado por PCR punto final. Carriles: 1) Marcador de peso molecular $1 \mathrm{~kb}, 2$ ) blanco de la reacción, 3) control negativo células Rm-sus no infectadas, 4) control positivo sangre infectada con la cepa MEX-31-096, 5 al 10) células Rm-sus infectadas con la cepa MEX-31-096 pases del 1 al 6 respectivamente.

Aunque A. marginale se mantuvo en cultivo por un tiempo limitado ( 5 pases durante 3 meses) estos resultados son valiosos para el desarrollo de nuestra investigación ya que demuestran que A. marginale (MEX-31-096) tiene la capacidad de infectar y propagarse en células Rmsus. Por otra parte, este sistema de cultivo abrirá nuevas perspectivas en el estudio de la anaplasmosis bovina ya que se podrá obtener estos microorganismos bajo condiciones controladas y en menor tiempo. Adicionalmente, estos microorganismos podrán ser utilizados para la generación de antígenos para su uso para el diagnóstico de la enfermedad $\mathrm{y}$, dado que la cepa posee una virulencia atenuada de forma natural es un candidato potencial para ser utilizados como inmunógeno.

\section{Conclusiones}

En este trabajo hemos reportado el primer abordaje para el establecimiento del cultivo in vitro de una cepa mexicana de A. marginale (MEX-31-096) utilizando como sustrato células embrionarias de garrapata Rm-sus. La propagación de A. marginale en células Rm-sus eliminará en gran medida el uso de animales para su replicación. Por otra parte, constituirá una herramienta para el estudio de las cepas y aislados mexicanos de A. marginale ya que facilitará la obtención de antígenos para el diagnóstico de la enfermedad reduciendo tiempo y costos. Asimismo, permitirá el desarrollo de estudios in vitro para el desarrollo de vacunas contra la anaplasmosis. 
Cobaxin-Cardenas, M; Aguilar Díaz, H.; Olivares Avelino, P.; Salinas-Estrella, E.; Preciado-de la Torre, J.; Quiroz-Castañeda, R.; Amaro-Estrada, I.; Cossío-Bayúgar, R. ; Rodríguez-Camarillo, S.

\section{Agradecimientos}

Este proyecto fue financiado por el fondo INIFAP (recursos fiscales) con número de SIGI 13341734501. El autor de correspondencia agradece a la Dra. Susan Noh por el entrenamiento técnico en el cultivo in vitro de A. marginale en la Universidad Estatal de Washington. Pullman, Washington y a PROCINORTE por el financiamiento otorgado para dicho entrenamiento.

\section{Referencias}

Baêta B.A., Ribeiro Carla C.D.U., Teixeira R.C. Cabezas-Cruz A., Passos Lygia M.F., Zweygarth E., y Fonseca A.H. (2015). Characterization of two strains of Anaplasma marginale isolated from cattle in Rio de Janeiro, Brazil, after propagation in tick cell culture. Ticks and Tick-borne Diseases. 6:(2), 141-145.

Bastos, C. V., Passos, L. M., Facury-Filho, E. J., Rabelo, E. M., De La Fuente, J., \& Ribeiro, M. F. B. (2010). Protection in the absence of exclusion between two Brazilian isolates of Anaplasma marginale in experimentally infected calves. The Veterinary Journal, 186(3), 374-378.

Blouin, E. F., Barbet, A. F., Yi, J., Kocan, K. M., \& Saliki, J. T. (2000). Establishment and characterization of an Oklahoma isolate of Anaplasma marginale. In cultured Ixodes scapularis cells. Veterinary parasitology, 87(4), 301-313.

Blouin, E. F., \& Kocan, K. M. (1998). Morphology and development of Anaplasma marginale (Rickettsiales: Anaplasmataceae) in cultured Ixodes scapularis (Acari: Ixodidae) cells. Journal of medical entomology, 35(5), 788-797.

Cossío-Bayugar, R., \& Miranda-Miranda, E. (2007). Heterologus gene expression in a cattle tick Rhipicephalus microplus embryonic cell culture. Journal of Animal and Veterinary Advances, 6(10), 1214-1218.

Esteves, E., Bastos, C. V., Zivkovic, Z., de La Fuente, J., Kocan, K., Blouin, E., ... \& Daffre, S. (2009). Propagation of a Brazilian isolate of Anaplasma marginale with appendage 
in a tick cell line (BME26) derived from Rhipicephalus (Boophilus) microplus. Veterinary parasitology, 161(1-2), 150-153.

Hammac, G. K., Ku, P. S., Galletti, M. F., Noh, S. M., Scoles, G. A., Palmer, G. H., \& Brayton, K. A. (2013). Protective immunity induced by immunization with a live, cultured Anaplasma marginale strain. Vaccine, 31(35), 3617-3622.

Kocan, K. M., De la Fuente, J., Guglielmone, A. A., \& Meléndez, R. D. (2003). Antigens and alternatives for control of Anaplasma marginale infection in cattle. Clinical microbiology reviews, 16(4), 698-712.

Lis, K., de Mera, I. G. F., Popara, M., Cabezas-Cruz, A., Ayllón, N., Zweygarth, E., ... \& Ribeiro, M. F. (2015). Molecular and immunological characterization of three strains of Anaplasma marginale grown in cultured tick cells. Ticks and tick-borne diseases, 6(4), 522-529.

Munderloh, U. G., Blouin, E. F., Kocan, K. M., Ge, N. L., Edwards, W. L., \& Kurtti, T. J. (1996). Establishment of the tick (Acari: Ixodidae)-borne cattle pathogen Anaplasma marginale (Rickettsiales: Anaplasmataceae) in tick cell culture. Journal of medical entomology, 33(4), 656-664.

Munderloh, U. G., Liu, Y., Wang, M., Chen, C., \& Kurtti, T. J. (1994). Establishment, maintenance and description of cell lines from the tick Ixodes scapularis. The Journal of parasitology, 80(4), 533-543.

Ortiz, M. Á. G., Torres, R. A., Salgado, G. H., Alarcón, G. J. C., \& Camarillo, S. D. R. (2000). Anaplasma marginale: diferentes grados de virulencia en dos aislados mexicanos. Veterinaria México, 31(2), 157-160.

Palmer, G. H., Knowles, D. P., Rodriguez, J. L., Gnad, D. P., Hollis, L. C., Marston, T., \& Brayton, K. A. (2004). Stochastic transmission of multiple genotypically distinct Anaplasma marginale strains in a herd with high prevalence of Anaplasma infection. Journal of Clinical Microbiology, 42(11), 5381-5384. 
Cobaxin-Cardenas, M; Aguilar Díaz, H.; Olivares Avelino, P.; Salinas-Estrella, E.; Preciado-de la Torre, J.; Quiroz-Castañeda, R.; Amaro-Estrada, I.; Cossío-Bayúgar, R.; Rodríguez-Camarillo, S.

Quiroz-Castañeda, R. E., Amaro-Estrada, I., \& Rodríguez-Camarillo, S. D. (2016). Anaplasma marginale: diversity, virulence, and vaccine landscape through a genomics approach. BioMed research international, 2016.

Rodríguez, S. D., Ortiz, M. Á. G., Ocampo, R. J., \& y Murguía, C. A. V. (2009). Molecular epidemiology of bovine anaplasmosis with a particular focus in Mexico. Infection, Genetics and Evolution, 9(6), 1092-1101.

Servicio Nacional de Sanidad, Inocuidad y Calidad Agroalimentaria. 2019. Situación Zoosanitaria en los Estados de la República Mexicana (3 de octubre de 2019). Consultado en https://www.gob.mx/senasica/documentos/informes-de-la-situacionzoosanitaria-nacional-2019?state=published el 18 de octubre de 2019.

Silveira, J. A., Silvestre, B. T., Bastos, C. V., \& Ribeiro, M. F. (2016). Isolation and attempted cultivation of an Anaplasma marginale strain from Brazilian brown brocket deer (Mazama gouazoubira, Fisher, 1814) in the tick cell line IDE8. Ticks and tickborne diseases, 7(6), 1102-1108.

Whitlock, B.J. (2014). Seroprevalence of bovine anaplasmosis in the southern US. The AABP Proceedings. Consultado

en http://foundation.aabp.org/research_proposal/2012_grant_Whitlock.pdf 